\title{
Spectral Feature Detection with sub-Nyquist Sampling for Wideband Spectrum Sensing
}

\author{
Eva Lagunas*, Member, IEEE, and Montse Nájar, Senior Member, IEEE.
}

\begin{abstract}
Compressive Sensing (CS) has been successfully applied to alleviate the sampling bottleneck in wideband spectrum sensing leveraging the sparsity described by the low spectral occupancy of the licensed radios. However, the existence of interferences emanating from low-regulated transmissions, which cannot be taken into account in the CS model because of their non-regulated nature, greatly degrade the identification of licensed activity. This paper presents a feature-based technique for primary user's spectrum identification with interference immunity which works with a reduced amount of data. The proposed method not only detects which frequencies are occupied by primary users' but also identifies the primary users' transmitted power. The basic strategy is to compare the $a$ priori known spectral shape of the primary user with the power spectral density of the received signal. This comparison is made in terms of autocorrelation by means of a correlation matching, thus avoiding the computation of the power spectral density of the received signal. The essence of the novel interference rejection mechanism lies in preserving the positive semidefinite character of the residual correlation, which is inserted by means of a weighted formulation of the $l_{1}$-minimization. Simulation results show the effectiveness of the technique for interference suppression and primary user detection.
\end{abstract}

Index Terms-Sub-Nyquist sampling, spectrum sensing, cognitive radio, compressive sensing.

\section{INTRODUCTION}

$\mathbf{S}$ PECTRUM sharing has gained a special attention in the research community for its promising results in improving the spectral efficiency [1]-[3]. The present regulation of the electromagnetic spectrum based on exclusive licensing of a particular band within a defined geographic region has been shown to be inefficient, with most of the allocated spectrum being underutilized [4]. The inefficient usage of the spectrum together with the ever-increasing demand of emerging services for more radio spectrum suggest that a transition to a more intelligent and flexible spectrum management regime is needed. These considerations have motivated the promotion of Cognitive Radio (CR) [5], [6]. CR resolves the problem of

E. Lagunas is with the Interdisciplinary Centre for Security, Reliability and Trust (SnT), University of Luxembourg (http://www.securityandtrust.lu), L-2721 Luxembourg. E-mail: eva.lagunas@uni.lu.

M. Nájar is with the Signal Theory and Communications Department, Universitat Politècnica de Catalunya, Barcelona, 08034 Spain. E-mail: montse.najar@upc.edu.

The research leading to these results has received funding from the Spanish Ministry of Science and Innovation (Ministerio de Ciencia e Innovación) under project TEC2011-29006-C03-02 (GRE3N-LINK-MAC), from the European Commission in the framework of the FP7 Network of Excellence in Wireless COMmunications NEWCOM\# (Grant agreement no. 318306) and from the Catalan Government under grant 2014-SGR-60.

Part of this work was submitted to IEEE International Conference on Acoustics, Speech, and Signal Processing (ICASSP), 2014.

${ }^{*}$ Corresponding author. limited spectral resources by enabling unlicensed (secondary) systems to opportunistically utilize the unused licensed (primary) bands. The task of accurately detecting the presence of licensed user is encompassed in spectrum sensing [7].

The traditional way for detecting holes in a wide-band spectrum is channel-by-channel sequential scanning [8], which might introduce large latency. Another possible way is to use an RF front-end with a bank of narrow band-pass filters. This approach solves the latency problem, since multiple channels are processed simultaneously. However, it is inefficient due to the numerous required RF components for the implementation. An alternative approach is to directly sense a wide frequency range at the same time, the so-called Wideband Spectrum Sensing (WSS) (see [9] and the reference therein). However, special attention should be paid to the wideband processing, which renders high-rate standard analog-to-digital converter (ADC) costly and even impractical. Clearly, the need to process very wide bandwidth represents the most critical challenge of WSS [10], [11].

In this context, Compressive Sensing (CS) [12], [13] has emerged as a promising signal processing technique to simultaneous sensing and compressing sparse signals thus allowing sampling rates significantly lower than Nyquist rate. As the wideband spectrum is inherently sparse due to the low percentage of spectrum occupancy (the motivating fact of dynamic spectrum management), CS becomes a promising technique to reduce the burden on the ADCs in WSS. CS was first apply to WSS in [14], where sub-Nyquist rate samples are utilized to detect and classify frequency bands via wavelet-based edge detector. The problem of sampling a signal at minimal rate and reconstruct the spectrum from the compressive measurements is discussed in [15]-[17]. Power spectrum estimation methods based on sub-Nyquist rate samples were presented in [18], [19], where the spectrum of the uncompressed signal is retrieved by concentrating on the autocorrelation function instead of the original signal itself.

All aforementioned approaches for CS applied to spectrum sensing fall in the class of blind techniques, i.e., they avoid the need for prior knowledge on the primary user. Classic examples of blind spectrum sensing techniques are energy detector [20], and the recently proposed eigenvalue-based detection [21]. The drawback though is that the blind nature of these techniques impedes discrimination between sources of received energy namely primary signal, noise and interference. In practical $\mathrm{CR}$, primary signals must be detected even with the presence of low-regulated transmissions from secondary systems. The existence of interferences emanating from lowregulated transmissions, which cannot be taken into account in 
the problem formulation because of their non-regulated nature, greatly degrades the identification of licensed activity [22].

Signals used in practical communication systems contain distinctive features that can be exploited for detection and that enable us to achieve a detection performance that substantially surpasses that of blind techniques. In category of non-blind sensing and when the reference signal is precisely known $a$ priori, matched filtering [10] is known to be the optimum method for detection. However, matched filtering cannot be directly applied to WSS because they make a single decision for the whole spectrum, impeding identification of individual spectrum opportunities.

This paper presents a feature-based technique for primary user's spectrum identification with interference immunity which works with a reduced amount of data. The proposed method detects which channels are occupied by primary users' and also identify the primary users transmission powers, thus providing ranging information (i.e., information about the distance between the primary user and the sensing device). The spectrum characteristics of the primary signals, which can be obtained by identifying its transmission technologies in public standards, are used as features. The basic strategy is to compare the a priori known spectral shape of the primary user with the power spectral density of the received signal. This comparison is made in terms of autocorrelation by means of a correlation matching, thus avoiding the computation of the Power Spectral Density (PSD) of the received signal. To do so, we devise an overcomplete dictionary [23] containing potential correlations for sparse representation of the primary user spectral support, thus allowing all possible hypothesis to be evaluated simultaneously in a global unified optimization framework. Extraction of the primary user frequency locations is then performed based on sparse signal recovery algorithms. The essence of the novel interference rejection mechanism lies in preserving the positive semidefinite character of the residual correlation, which is inserted by means of a weighting procedure for sparse approximation. It is noteworthy that the occupied channels are directly detected from the sample autocorrelation matrix avoiding the complete signal and spectrum reconstruction.

In this context, the contribution of the paper can be summarized as follows:

- We derive a CS-based correlation matching approach for identification of licensed users in the context of CR. The main advantage of the proposed approach with respect to the spectral feature detection presented in [24] is the robustness to interference, which is achieved in this paper by preserving the positive semi-definite condition on the residual correlation. Moreover, [24] did not deal with a reduced data volume.

- It is worth noting that we do not aim for reconstructing the power spectrum from compressive measurements but rather for primary's carrier frequency recovery, which does not require perfect signal reconstruction or approximation. Since the spectral shape of the licensed holder is assumed to be known, the licensed spectral support can be estimated as a follow on step of the proposed detector. - The correlation matching strategy followed in this paper was shown in [24], [25] to be less costly than the cyclostationary detector [26] in terms of computational complexity. Moreover, spectral feature detectors exploit unique spectral pattern of the primary signal, which goes a step further than periodicity in the mean and autocorrelation.

- A thorough performance assessment is also given, and the results show the superiority and efficiency of the proposed detector over the existing approaches in interference suppression and primary user detection performance.

This work extends previous authors' publications [27]-[29]. In [27], we proposed a spectral scan with a particular shape without caring about the high-speed sampling requirement. In [28], [29], the sparsity of the primary signals sent out over the spectrum was used to alleviate the sampling burden but not imposed on the primary user detection. In this paper, we take advantage of the sparsity described by the licensed users spectral support not only to alleviate the sampling bottleneck but also to increase the accuracy of the detections by using sparsity-based recovery methods.

This paper is organized as follows. Section II reviews the spectrum sensing problem. Section III introduces the second order based spectrum sensing and motivates the use of CS. Section IV reviews the non-uniform periodic sub-Nyquist sampling notation. The correlation-matching based licensed user identification is described in Section V. In Section VI, we demonstrate, via simulation, the performance of the proposed method. Conclusions are drawn in Section VII.

Notation: Throughout the paper, scalars are denoted by nonboldface type, vectors by boldface lowercase letters and matrices by boldface uppercase letters. Superscripts $(\cdot)^{T},(\cdot)^{*}$ and $(\cdot)^{H}$ denote transpose, complex conjugate and complex conjugate transpose, respectively. $\|\mathbf{a}\|_{l_{p}}$ denote the $l_{p}$-norm of vector a, i.e., $\|\mathbf{a}\|_{l_{p}}=\left(\sum_{i=1}^{n}\left|a_{i}\right|^{p}\right)^{1 / p}$. The symbol $\odot$ denotes the elementwise product and $\operatorname{kron}(\cdot, \cdot)$ denotes the Kronecker product. The modulus and the usual inner product are denoted by $|\cdot|$ and $\langle\cdot, \cdot\rangle$, respectively.

\section{Problem Statement}

Our goal is to decide whether a given frequency band is occupied by a primary user or not using a sub-Nyquist sampling alternative suitable for sparse signals. Let us denote $x(t)$ the wideband signal representing the superposition of different primary services in a CR network. Signal $x(t)$ is assumed to be sparse multiband signal, i.e, a bandlimited, continuous-time, squared integrable signal that has all of its energy concentrated in a small number of disjoint frequency bands. In other words, its spectral support has Lebesgue measure small relative to the overall signal bandwidth [30]. Denoting the Fourier transform of $x(t)$ as $X(f)$, the spectral support of the multiband signal $x(t)$, denoted as $\mathcal{F} \subset\left[0, f_{\max }\right]$, namely $X(f)=0, f \notin \mathcal{F}$, is restricted to have its signal energy distributed on no more than $N_{b}$ disjoint bands in $\mathcal{F}$ :

$$
\mathcal{F}=\bigcup_{i=1}^{N_{b}}\left[a_{i}, b_{i}\right)
$$


where $\left[a_{i}, b_{i}\right), i=1, \ldots, N_{b}$, represent the edges of each band. The spectral occupancy $\Omega$ is defined as,

$$
\Omega=\frac{\lambda(F)}{f_{\max }} \quad 0 \leq \Omega \leq 1
$$

where $\lambda(F)$ is the Lebesgue measure of the frequency set $F$, which, in this particular case, is equal to $\sum_{i=1}^{N_{b}}\left(b_{i}-a_{i}\right)$. In the spectrum sensing framework, the spectral support $F$ is unknown but the total bandwidth under study is assumed to be sparse, i.e., $\Omega<<1$.

The basic idea of $\mathrm{CR}$ is spectral reusing or spectrum sharing, which allows the unlicensed users to communicate over licensed spectrum when the licensed holders are not fully utilizing it. Thus, the received signal $y(t)$ consists on the superposition of multiple primary and secondary (interference) services. Let us write the received signal as,

$$
y(t)=\sum_{k=1}^{K} x\left(t, \omega_{k}\right)+i(t)+\eta(t)
$$

where $K$ denotes the number of primary users. The analytic representation of the $k$-th primary user is denoted as $x\left(t, \omega_{k}\right)$, which is given by,

$$
x\left(t, \omega_{k}\right)=a_{k}(t) e^{j\left(\omega_{0}+\omega_{k}\right) t}
$$

where $a_{k}(t)$ indicates the complex envelope of the source and $\omega_{k}$ denotes the baseband frequency of the source with respect to the center frequency of the band under scrutiny, $\omega_{0}$. Note that, in (4), we aimed to separate the frequency location information given by $e^{j\left(\omega_{0}+\omega_{k}\right) t}$ from the symbol modulation information given by $a_{k}(t)$. For the sake of simple notation, we are assuming linear propagation channel with no distortion. However, the robustness in front of a frequency selective channel, instead of flat fading, have also been studied using computer simulations.

The non-regulated activity of the spectrum is denoted in (3) as $i(t)$ and henceforth is considered interference. The problem of determining whether a given frequency is occupied or not by a licensed radio can be modeled into a binary hypothesis test,

$$
y(t)= \begin{cases}i(t)+\eta(t) & \left(H_{0}\right) \\ x(t)+i(t)+\eta(t) & \left(H_{1}\right)\end{cases}
$$

where $\left(H_{0}\right)$ stands for the absence of primary signal and $\left(H_{1}\right)$ represents the presence of a primary signal in the frequency channel under study. $\eta(t)$ is $\mathrm{AWGN}, \mathcal{C N}\left(0, \sigma_{\eta}^{2}\right)$. The interference $i(t)$ is independent of the noise and primary signal, and we assume that its spectral shape is different from that of the primary. In practice, the secondary user is rarely going to use the same modulation as the primary [14], [24], [25], [29]. Moreover, the secondary user generally transmits at a lower rate than the primary in order to not disturb the quality of service of the primary user. Therefore, and as a baseline, it is assumed throughout this paper that the interference has bandwidth smaller than that of the primary. The presence of unknown interference, which has not been addressed in conventional spectrum sensing publications, adds additional challenges.

\section{Spectrum Sensing based on Second Order STATISTICS}

Energy detection is the most widely used spectrum sensing technique since it is simple to implement and does not require any prior information about the primary signal [31]. However, the prevalence of digital modulations in common primary users motivate more sophisticated and creative spectrum sensing techniques.

For the purpose of the present work, let us define $r_{c}(\tau)$ as the baseband conventional autocorrelation function (CAF) of the reference signal $x_{c}(t)$. The expression of $r_{c}(\tau)$ is linked to the PSD of $x_{c}(t)$, which, for linearly modulated signals, depends mainly on the transmission rate and the modulation pulse. The latter comes from the fact that the average power spectral density of the analytic signal of $x_{c}(t)$, for positive frequencies, memoryless channel and zero-mean signal is given by,

$$
S_{c m}^{+}(\omega)=\gamma R_{b}\left|P\left(\omega-\omega_{0}\right)\right|^{2}
$$

where $\gamma$ is the actual power level measured at the sensing station, $\omega_{0}$ is the carrier frequency, $R_{b}$ is the baud rate and $P(\omega)$ the spectrum of the modulation pulse which satisfies $\frac{1}{2 \pi} \int|P(\omega)|^{2} \mathrm{~d} \omega=1$. Therefore, the elements of the reference CAF are given by the discrete Fourier transform of $S_{c m}^{+}(\omega) / \gamma$. The aforementioned characteristics of $x_{c}(t)$ required for the computation of $r_{c}(\tau)$ can be analytically extracted from physical layer standardization of primary services.

Clearly, the proposed algorithm is introduced here to detect a specific primary system which is determined by the candidate autocorrelation $r_{c}(\tau)$. It is noteworthy, however, that the algorithm can be straightforward extended to detect different kind of users, as long as their spectrum characteristics are known.

Let $\mathcal{B}$ denote the set of possible primary users' frequency locations and let $\left\{\omega_{m}\right\}_{m=0}^{M-1}$ denote a grid that covers $\mathcal{B}$. Assuming that the true location of the primary users lie on the grid, the corresponding model for the CAF of the received signal $r_{y}(\tau)$ is given by,

$$
r_{y}(\tau)=\sum_{m=1}^{M} p\left(\omega_{m}\right) \cdot r_{c m}\left(\tau, \omega_{m}\right)+r_{i}(\tau)+r_{\eta}(\tau)
$$

where $r_{i}(\tau)$ and $r_{\eta}(\tau)$ denote the CAFs of the interference and the noise, respectively, $p\left(\omega_{m}\right)$ is the primary user' transmitted power corresponding to $\omega_{m}$, and $r_{c m}\left(\tau, \omega_{m}\right)$ denotes the candidate CAF modulated at the specific carrier frequency $\omega_{m}$.

As indicated by extensive spectrum occupancy measurements campaigns [32] [33], numerous spectrum bands are vacant although licenses have been issued by the regulatory agencies, suggesting that the frequency spectrum is sparse. The previous formulation of the location problem begs for the use of basic ideas from the area of CS.

Note that only a small number of primary users exists and therefore only few grid points that covers $\mathcal{B}$ are occupied, i.e., denoting $K$ the number of primary users present in $y(t), K<<M$. Indeed, as the value of $M$ increases with respect to $K, \mathcal{B}$ becomes more and more sparsely represented. Thus, the model presented in (7) implicitly assume the use of 
overcomplete dictionary [23] of candidate CAFs, in which, ideally, only few of them are present in the received CAF. Therefore, the sparse vector defined by,

$$
\mathbf{p}=\left[\begin{array}{llll}
p\left(\omega_{0}\right) & p\left(\omega_{1}\right) & \cdots & p\left(\omega_{M-1}\right)
\end{array}\right]^{T}
$$

can be viewed as the output of an indicator function, whose elements different from zero directly indicate the carrier frequencies $\omega$ where primary signals are present. The sparse nature of the vector $\mathbf{p}$ motivates the design of novel detection strategies based on the CS theory.

\section{COMPRESSIVE SAMPLING}

The abbreviation CS stands for Compressive Sensing or Compressive Sampling, which is the name given to the subNyquist sampling techniques that exploit sparse structure in data. Compared with traditional Nyquist sampling theory, the number of samples required in CS can be made much smaller by exploiting the signal sparsity property exhibited in a certain domain. Periodic Nonuniform Sampling (PNS) is a popular approach among those techniques, specially when only covariance information is of interest [34]. In this paper we consider the sub-Nyquist PNS strategy known as multicoset (MC) sampling [35], [36]. Given the received multiband signal $y(t)$, the MC samples are obtained at the time instants,

$$
t_{i}(n)=\left(n L+c_{i}\right) T
$$

where $L>0$ is a suitable integer, $i=1,2, \ldots, \kappa$ and $n \in \mathbb{Z}$. The set $\left\{c_{i}\right\}$ contains $\kappa$ distinct integers chosen from $\{0,1, \ldots, L-1\}$. The reader can notice that the MC sampling process can be viewed as a classical Nyquist sampling followed by a block that discards all but $\kappa$ samples in every block of $L$ samples periodically. The samples which are not thrown away are specified by the set $\left\{c_{i}\right\}$. Thus, a sequence (or coset, hence the name of the method) of equally-spaced samples is obtained for each $c_{i}$. The period of each one of these sequences is equal to $L T$. Therefore, one possible implementation consists of $\kappa$ parallel ADCs, each working uniformly with period $L T$.

One limitation of multi-coset sampling is maintaining accurate time delays between the ADCs of different cosets. Here, we assume perfect synchronization between multi-coset sampling branches and we refer the reader to [37] for details concerning the asynchronous multi-rate wideband sensing.

The complete observation consists of a data record of $N_{f}$ blocks of $\kappa$ non-uniform samples notated as $\mathbf{y}_{f}$. Thus, the notation can be compacted in $\mathbf{Y}$ as follows,

$$
\mathbf{Y}=\left[\begin{array}{lll}
\mathbf{y}_{1} & \ldots & \mathbf{y}_{N_{f}}
\end{array}\right]
$$

In order to relate the acquired samples with the original Nyquist-sampled signal, let us consider $\mathbf{z}_{f}$ as the $f$-th block of $L$ uniform Nyquist samples of $y(t)$,

$$
\mathbf{z}_{f}=\left[\begin{array}{lll}
y\left(t_{1}^{f}\right) & \ldots & y\left(t_{L}^{f}\right)
\end{array}\right]^{T}
$$

where $t_{n}^{f}=(f L+n) T$. Thus, the relation between the Nyquist samples and the sub-Nyquist samples is given by,

$$
\mathbf{y}_{f}=\mathbf{\Phi} \mathbf{z}_{f}
$$

where $\boldsymbol{\Phi}$ is a matrix that non-uniformly selects $\kappa$ samples of $\mathbf{z}_{m}(\kappa<L)$. This matrix $\boldsymbol{\Phi}$ is obtained by non-uniformly selecting $\kappa$ rows of the identity matrix $\mathbf{I}_{L}$.

\section{Compressive Spectrum Sensing Via Spectral MATCHING}

Let $\left\{R_{y}(k)\right\}$ denotes the correlations of the Nyquistsampled signal $\{y(n)\}$ and $\mathbf{R}_{y}$ be the $L \times L$ correlation matrix (i.e. $\left[\mathbf{R}_{y}\right]_{i j}=R_{y}(j-i)$ ). For the purpose of the present work, let us define $\left\{R_{c}(k)\right\}$ as the correlations of the Nyquistsampled candidate signal $\left\{x_{c}(n)\right\}$. Following the notation of (12), the correlation matrix $\hat{\mathbf{R}}_{y} \in \mathbb{C}^{\kappa \times \kappa}$ can be obtained as,

$$
\hat{\mathbf{R}}_{y}=\frac{1}{N_{f}} \sum_{f=1}^{N_{f}} \mathbf{y}_{f} \mathbf{y}_{f}^{H}=\boldsymbol{\Phi}\left[\frac{1}{N_{f}} \sum_{f=1}^{N_{f}} \mathbf{z}_{f} \mathbf{z}_{f}^{H}\right] \boldsymbol{\Phi}^{H}
$$

Conventional spectrum estimation techniques try to estimate how much power coming from a single pure frequency is contained in $\hat{\mathbf{R}}_{y}$. The CAF of a pure tone is given by $\mathbf{e}\left(\omega_{m}\right) \mathbf{e}^{H}\left(\omega_{m}\right)$, where $\mathbf{e}\left(\omega_{m}\right) \in \mathbb{C}^{L \times 1}$ is the frequency steering vector defined as,

$$
\mathbf{e}\left(\omega_{m}\right)=\left[\begin{array}{llll}
1 & e^{j \omega_{m}} & \ldots & e^{j(L-1) \omega_{m}}
\end{array}\right]^{T}
$$

In this paper, we would like to design a spectral analyzer whose impulse response is not the single pure frequency but a predetermined spectral shape. In order to obtain the frequency location of a particular spectral shape, the baseband reference autocorrelation $\mathbf{R}_{c} \in \mathbb{R}^{L \times L}$ (extracted from spectral features of the primary user) is modulated by a rank-one matrix formed by the steering frequency vector at the sensed frequency $\omega_{m}$ as follows,

$$
\mathbf{R}_{c m}\left(\omega_{m}\right)=\left[\mathbf{R}_{c} \odot \mathbf{e}\left(\omega_{m}\right) \mathbf{e}^{H}\left(\omega_{m}\right)\right]
$$

where $\odot$ denotes the elementwise product of two matrices. The resulting modulated candidate correlation $\mathbf{R}_{c m}\left(\omega_{m}\right) \in \mathbb{C}^{L \times L}$ must be compressed in the same way we did for the data, i.e., using the same sampling matrix $\boldsymbol{\Phi}$, as follows,

$$
\hat{\mathbf{R}}_{c m}\left(\omega_{m}\right)=\boldsymbol{\Phi} \mathbf{R}_{c m}\left(\omega_{m}\right) \boldsymbol{\Phi}^{H}
$$

According to this notation, the corresponding model for the data autocorrelation matrix defined in (13) is given by,

$$
\hat{\mathbf{R}}_{y}=\sum_{m=1}^{M} p\left(\omega_{m}\right) \hat{\mathbf{R}}_{c m}\left(\omega_{m}\right)+\hat{\mathbf{R}}_{\boldsymbol{\epsilon}}
$$

where $\hat{\mathbf{R}}_{\epsilon}$ encompasses the imperfections of the model, including interference and noise.

The sparse nature of vector $\mathbf{p}$, introduced in (8), can be exploited to avoid the tedious channel by channel scanning process. To this end, the model in (17) can be conveniently rewritten into a sparse notation as follows,

$$
\hat{\mathbf{r}}_{y}=\operatorname{kron}(\boldsymbol{\Phi}, \boldsymbol{\Phi}) \mathbf{B S p}+\boldsymbol{\epsilon}=\mathbf{A p}+\boldsymbol{\epsilon}
$$

where $\hat{\mathbf{r}}_{y}$ is a $\kappa^{2} \times 1$ vector formed by the concatenation of the columns of $\hat{\mathbf{R}}_{y}$. From now on, to clarify notation, the concatenation of columns will be denoted with the operator $\operatorname{vec}(\cdot)$. Therefore, $\hat{\mathbf{r}}_{y}=\operatorname{vec}\left(\hat{\mathbf{R}}_{y}\right)$. B contains the spectral 


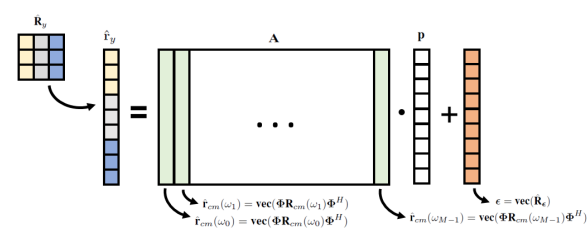

Fig. 1. Scheme of the sparse notation of (18)

information of the primary signals and is defined as $\operatorname{diag}\left(\mathbf{r}_{c}\right)$ where $\mathbf{r}_{c}$ is $\operatorname{vec}\left(\mathbf{R}_{c}\right)$. Matrix $\mathbf{S}$ defines the frequency scanning grid,

$$
\mathbf{S}=\left[\begin{array}{llll}
\mathbf{s}\left(\omega_{0}\right) & \mathbf{s}\left(\omega_{1}\right) & \cdots & \mathbf{s}\left(\omega_{M-1}\right)
\end{array}\right]
$$

where $\mathbf{s}\left(\omega_{m}\right)$ is defined as $\operatorname{vec}\left(\mathbf{e}\left(\omega_{m}\right) \mathbf{e}^{H}\left(\omega_{m}\right)\right)$. The variable $\boldsymbol{\epsilon}$ encompasses the contribution of $\operatorname{vec}\left(\hat{\mathbf{R}}_{\boldsymbol{\epsilon}}\right)$. A greater insight can be obtained from Fig. 1. It is worth highlighting that the overcomplete dictionary leading to sparse representation is given in (18) by the matrix BS, whose columns contain the information regarding the different candidate CAFs previously introduced in (7). The term $\operatorname{kron}(\boldsymbol{\Phi}, \boldsymbol{\Phi})$ in (18) establishes which entries of each of the non-compressive candidate correlation are available after digitizing the signal according to the sampling pattern $\boldsymbol{\Phi}$ introduced in (12).

Since $\kappa<L$, there are infinitely many solutions to the following problem,

$$
\min _{\mathbf{p} \geq 0}\left\|\hat{\mathbf{r}}_{y}-\mathbf{A p}\right\|_{l_{2}}
$$

Among all the solutions of (20), we are interested in the solution that meets the following requirements: (1) p must be sparse, and (2) ( $\left.\hat{\mathbf{r}}_{y}-\mathbf{A p}\right)$ must provide a residual positive semidefinite correlation. The latter is justify as follows. Although the spectral shapes of the license-holder user is assumed to be different from that of the opportunistic users, they can be quite similar. To avoid excessive false alarm rate and strengthen the interference rejection capabilities of the proposed spectral feature detector, the residual correlation is enforced to be positive semidefinite. In the following section, we justify the importance of this restriction.

\section{A. Enforcing positive semidefinite residual correlation}

Correlation matrices are hermitian positive semi-definite matrices by definition and they lie in a convex cone [38]. Thus, the difference between $\hat{\mathbf{R}}_{y}$ and the compressed modulated candidate matrix $p\left(\omega_{m}\right) \hat{\mathbf{R}}_{c m}\left(\omega_{m}\right)$ must lie in the surface of the cone too, since it is still a correlation matrix. In other words, the residual matrix must be positive semi-definite. This is,

$$
\hat{\mathbf{R}}_{y}-p\left(\omega_{m}\right) \hat{\mathbf{R}}_{c m}\left(\omega_{m}\right) \succeq 0
$$

Note that the choice of conventional Euclidean distance between matrices is not appropriate here. The points on the cone are connected by exponential paths and only locally resemble a flat Euclidean space. The Euclidean distance (Frobenius norm) is well suited for linear spaces, and not for the cone-like spaces. In other words, it does not guarantee that the residual matrix $\hat{\mathbf{R}}_{y}-p\left(\omega_{m}\right) \hat{\mathbf{R}}_{c m}\left(\omega_{m}\right)$ is a positive semi-definite matrix.
Therefore, the problem can be formulated as follows,

$$
\begin{array}{rl}
\max _{p\left(\omega_{m}\right) \geq 0} & p\left(\omega_{m}\right) \\
\text { s.t. } & \hat{\mathbf{R}}_{y}-p\left(\omega_{m}\right) \hat{\mathbf{R}}_{c m}\left(\omega_{m}\right) \succeq 0
\end{array}
$$

This is, the maximum $p\left(\omega_{m}\right)$ that guarantees the difference between the data autocorrelation matrix and the candidate autocorrelation matrix remains positive semi-definite. If $\hat{\mathbf{R}}_{y}-p\left(\omega_{m}\right) \hat{\mathbf{R}}_{c m}\left(\omega_{m}\right)$ is positive semidefinite, $\mathbf{I}-p\left(\omega_{m}\right) \hat{\mathbf{R}}_{y}^{-1} \hat{\mathbf{R}}_{c m}\left(\omega_{m}\right)$ must be too. Thus, using the Eigen-Decomposition of $\hat{\mathbf{R}}_{y}^{-1} \hat{\mathbf{R}}_{c m}\left(\omega_{m}\right)$ defined by $\mathbf{U}\left(\omega_{m}\right) \boldsymbol{\Lambda}\left(\omega_{m}\right) \mathbf{U}^{H}\left(\omega_{m}\right)$,

$$
\begin{array}{ll}
\mathbf{I}-p\left(\omega_{m}\right) \mathbf{U}\left(\omega_{m}\right) \boldsymbol{\Lambda}\left(\omega_{m}\right) \mathbf{U}^{H}\left(\omega_{m}\right) & \succeq 0 \Rightarrow \\
\mathbf{I}-p\left(\omega_{m}\right) \boldsymbol{\Lambda}\left(\omega_{m}\right) & \succeq 0
\end{array}
$$

Eqn. (23) defines $\kappa$ different constraints,

$$
1-p\left(\omega_{m}\right) \lambda_{q}\left(\omega_{m}\right) \geq 0 \quad q=1, \ldots, \kappa
$$

where $\lambda_{1}\left(\omega_{m}\right) \geq \lambda_{2}\left(\omega_{m}\right) \geq \ldots \geq \lambda_{\kappa}\left(\omega_{m}\right)$ are the eigenvalues contained in the $\boldsymbol{\Lambda}\left(\omega_{m}\right)$. Note that if (24) is satisfied for $q=1$, it is satisfied $\forall q$. Thus, the restriction that always ensures positive semi-definite residual correlation at each scanned parameter $\omega_{m}$ is given by,

$$
p\left(\omega_{m}\right) \leq \lambda_{1}^{-1}\left(\omega_{m}\right) \quad m=0, \ldots, M-1
$$

Note that the values $\lambda_{1}^{-1}\left(\omega_{m}\right), m=0, \ldots, M-1$, work as upperbounds on the estimated power of the desired signal. In the next section we show how to impose the positive semidefinite restriction together with the sparsity constraint.

\section{B. Imposing sparsity constraint}

The sparsity restriction is usually imposed by adding a $l_{1}$ norm constraint. In other words, among all vectors $\mathbf{p}$ consistent with the data, we pick that whose coefficient sequence has minimal $l_{1}$-norm,

$$
\min _{\mathbf{p} \geq 0}\|\mathbf{p}\|_{l_{1}} \quad \text { s.t. } \quad \hat{\mathbf{r}}_{y} \approx \mathbf{A p}
$$

where $\|\mathbf{p}\|_{l_{1}}=\sum_{m=0}^{M-1} p\left(\omega_{m}\right)$. The use of the $l_{1}$-norm, however, has been shown to heavily penalize large coefficients to the detriment of smaller coefficients [39]. To address this magnitude dependence, weighted $l_{1}$-norm have been proposed to democratically penalize nonzero entries. Let us consider the following weighted $l_{1}$-norm,

$$
\sum_{m=0}^{M-1} w_{m} \cdot p\left(\omega_{m}\right) \leq \alpha
$$

where $w_{0}, \ldots, w_{M-1}$ are positive weights. Note that the values of $p\left(\omega_{m}\right)$ must be greater than or equal to zero and, thus, the absolute value is removed for simplicity. The value of $\alpha$ depends on the chosen weights. One way to rectify the dependence on magnitude of the $l_{1}$-norm is to enforce each product $w_{m} \cdot p\left(\omega_{m}\right)$ be equal to 1 . Thus, the weights must be estimates of the inverse power corresponding to the primary users present in the band under scrutiny. Ideally,

$$
w\left(\omega_{m}\right)=\left\{\begin{array}{lll}
\frac{1}{p\left(\omega_{k}\right)} & \text { if } \omega_{m}=\omega_{k} & \left(H_{1}\right) \\
0 & \text { otherwise } & \left(H_{0}\right)
\end{array}\right.
$$


Interestingly, initial estimates of the powers can be obtained from the upperbound defined in (25),

$$
w_{m}=\lambda_{1}\left(\omega_{m}\right) \quad m=0, \ldots, M-1
$$

With the weights defined in (29), the value of $\alpha$ in (27) is approximately equal to the number of primary users present in the band under scrutiny, $K$, which determines the sparsity level of vector p. The sparsity level $K$ plays a fundamental role in solving the sparse vector recovery problem,

$$
\min _{\mathbf{p} \geq 0}\|\mathbf{W} \mathbf{p}\|_{l_{1}} \quad \text { s.t. } \quad \hat{\mathbf{r}}_{y} \approx \mathbf{A p}
$$

where $\mathbf{W} \in \mathbb{R}^{M \times M}$ is defined as $\mathbf{W}=\operatorname{diag}(\mathbf{w})$, being $\mathbf{w}=\left[\begin{array}{lll}w_{0} & \cdots & w_{M-1}\end{array}\right]^{T}$. For example, many iterative algorithms such as Matching Pursuit [40] typically run for $K$ iterations or the regularization parameter of Least Absolute Shrinkage and Selection Operator (LASSO) [41] is examined over a limited range of values which is a function of $K$. Moreover, the value of $K$ is used to estimate the minimum number of measurements required to perfectly recover the original signal. For instance, for very specific models such as the Gaussian sensing matrices, it is known that on the order of $\kappa=C \cdot 2 K \log (L / 2 K))$ samples are sufficient for stable recovery, where $C$ refers to a positive, universal constant [42]. Anyhow, it is clear that $K$ plays an important theoretical role in many aspects of CS. However, it is typically unknown in practice.

Current literature assume that the CS model (18) is either exact or corrupted by AWGN or bounded noise, and use these assumptions to establish a stopping criterion for greedy pursuit methods and/or to derive input parameters for the convex optimization. If these parameters are set incorrectly, the quality of the reconstructed signal significantly decreases [43]. Here, we face an even more complex problem in which the compressive measurements capture the entire signal space, which includes undesired interference. There are two main strategies for dealing with interference assuming prior knowledge on some characteristics of the interference signal, namely avoiding and canceling methods [44]-[46]. Having access to information about the corrupted measurements, however, is not always possible in practical applications. In the next section we will show how to overcome this drawback.

\section{Sparse Signal Recovery}

When the CS model is only corrupted by noise, there are two well-established recovery methods. One is $l_{1}$-norm minimization under $l_{2}$ constraint on the residual. In the former, the $l_{2}$-norm of the residual is upperbounded by a constant derived from the noise power. The second alternative relaxes the constraint on the reconstruction error with a regularization or threshold parameter which is establish using information about the noise magnitude [13], [47], [48]. A family of iterative algorithms [49] were shown to provide the same reconstruction accuracy, generally with less computational complexity. Consequently, iterative algorithms become more preferable than directly solving the optimization problem. Finding robust stopping criteria in iterative algorithms is a long standing problem. Some relevant stopping criteria are [50]:

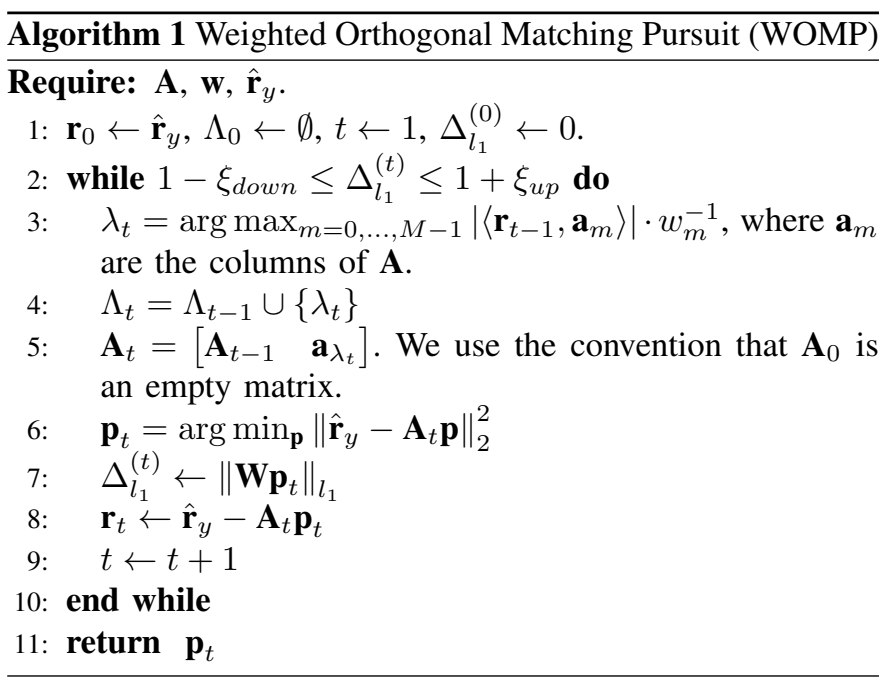

- Stop the algorithm after a fixed number of iterations.

- Wait until the $l_{2}$-norm of the residual declines to a certain level.

- Halt the algorithm when the maximum total correlation between an atom (column of $\mathbf{A}$ ) and the residual drops below a threshold.

Current iterative reconstruction algorithms generally assume a prior knowledge of the error variance and/or the signal sparsity to establish a stopping criterion. In particular, the number of iterations is linked with the sparsity of the signal to be recovered, which, in the problem at hand is given by $K$ (the number of primary users operating in the band under scrutiny). However, the value of $K$ is typically unknown in practice. There exist some works that try to overcome this issue. In [51], an estimate of the sparsity level is obtained by dividing the data set into a training/estimation set. In [52], an algorithm is proposed to estimate an approximation of the sparsity level. In the present work, we do not assume any prior information about the sparsity and neither about the noise/interference level. In essence, we introduced a heuristic stopping rule based on comparing consecutive weighted $l_{1}$ norms which can be viewed as a sparsity level estimator. In particular, we define a stopping criterion for the Weighted Orthogonal Matching Pursuit (WOMP) [53]. In [53], the WOMP algorithm is assumed to work in a noise-free model. Hence, the algorithm is halted when the energy of the residual generated by the greedy algorithm is close to zero. The aforementioned stopping criterion must be modified to cope with CS models contaminated with interference signals.

The proposed modification of WOMP is described with detail in Algorithm 1. At each iteration, the greedy selection chooses the atom that is most strongly correlated with the residual part of the signal, i.e., the atom that minimizes $\left\|\hat{\mathbf{r}}_{y}-\mathbf{A p}\right\|_{l_{2}}$. Denoting the columns of $\mathbf{A}$ as $\mathbf{a}_{m}$, the aforementioned selection at the $t$-th iteration is done as follows,

$$
\lambda_{t}=\arg \max _{m=0, \ldots, M-1}\left|\left\langle\mathbf{r}_{t-1}, \mathbf{a}_{m}\right\rangle\right| \cdot w_{m}^{-1}
$$

where $\mathbf{r}_{t-1}$ stands for the residual, which is initialized with $\mathbf{r}_{0}=\hat{\mathbf{r}}_{y}$. The weights defined in (29) impose that the licensedholder users are selected before the interference. This is 
because the value of $w_{m}^{-1}$ is expected to be ideally zero at the frequency bands that are not occupied by the licensed users. Let us define $\Delta_{l_{1}}^{(t)}$ the increment of weighted $l_{1}$-norm at iteration $t$ defined as,

$$
\Delta_{l_{1}}^{(t)}=\sum_{m=0}^{M-1} w_{m} \cdot p^{(t)}\left(\omega_{m}\right)-\sum_{m=0}^{M-1} w_{m} \cdot p^{(t-1)}\left(\omega_{m}\right)
$$

where $\mathbf{p}^{(t)}$ denotes the estimated sparse vector at iteration $t$. Interestingly, when a licensed-holder user is selected at iteration $t, \Delta_{l_{1}}^{(t)}$ is expected to be ideally one. Consequently, after $K$ iterations, the weighted $l_{1}$-norm of $\mathbf{p}^{(K)}$ is expected to be equal to $K$ and the residual is expected to contain noise plus interference. We would like the algorithm to stop here, since all primary users have been captured in the solution. If we keep running the algorithm, the next selected atom will probably correspond to an interference signal and, as a consequence, $\Delta_{l_{1}}^{(K+1)}$ will be different than one (ideally infinity). This is again because $w_{m} \rightarrow \infty$ at the frequency bands that are not occupied by the licensed users. So, we propose to stop the iterations when $\Delta_{l_{1}}^{(t)}$ does not fall into the following interval,

$$
1-\xi_{\text {down }} \leq \Delta_{l_{1}}^{(t)} \leq 1+\xi_{\text {up }}
$$

The values of $\xi_{\text {down }} \in[0,1)$ and $\xi_{u p} \in[0, \infty)$ determine the detector performance as we will show in the next Section. Some hints on how to set the parameters $\xi_{\text {down }}$ and $\xi_{\text {up }}$ are given below:

- $\xi_{\text {down }}$ : It is linked to the noise rejection capability. Therefore, it can be determined if some information regarding the noise statistics or noise magnitude is available. This problem is related to the threshold selection for Energy Detection (ED) techniques, where the noise power needs to be estimated if not known [54] [55].

- $\xi_{u p}$ : It is linked to the interference rejection capability and some numerical results on its dependence with the probability of false alarm are provided in the Section VI. The value of $\xi_{u p}$ can be estimated based on the similarity of the primary and secondary users spectral shapes. Depending on the application, we may assume a worst case scenario (i.e. a interference using similar spectral shape than that of the primary) and set the value of $\xi_{u p}$ that ensures a fixed number of false detections. Depending on the scenario, we can assume some feedback from the primary in terms of Quality of Service degradation and adapt the value of $\xi_{u p}$ accordingly (see [56] and the reference therein).

\section{Vi. Simulation Results}

In this section, we evaluate the performance of the proposed scheme using synthesized data. The spectral band under scrutiny has bandwidth equal to $f_{\max }=20 \mathrm{MHz}$. The size of the observation $\mathbf{z}_{f}$ is $L=33$ samples. The sampling rates of $\mathbf{y}_{f}$ and $\mathbf{z}_{f}$ are related through the compression rate $\rho=\frac{\kappa}{L}$. To strictly focus on the performance behavior due to compression and remove the effect of insufficient data records, the size of the compressed observations is forced to be the same for any compression rate. Therefore, we set $N_{f}=2 L \delta \rho^{-1}$ where $\delta$
TABLE I

SIMULATION PARAMETERS

\begin{tabular}{|c|c|c|c|c|}
\hline $\boldsymbol{\rho}$ & $\mathbf{1}$ & $\mathbf{0 . 7 6}$ & $\mathbf{0 . 5 2}$ & $\mathbf{0 . 2 4}$ \\
\hline $\boldsymbol{\kappa}$ & 33 & 25 & 17 & 8 \\
\hline$N_{f}$ & 660 & 871 & 1281 & 2723 \\
\hline
\end{tabular}

is a constant (in the following results $\delta=10$ ). Thus, for a high compression rate, the estimator takes samples for a larger period of time. Note that the value of $M$ determines the grid density of the spectrum to be scanned. Increasing $M$ makes the grid finer, but it also increases the computational complexity. On the other hand, making the grid too rough might introduce substantial bias into the estimates. Unless otherwise noted, the grid resolution for this section is $\Delta_{\omega}=100 \mathrm{kHz}$. The simulation parameters are summarized in Table I.

To test the ability of the proposed sparse spectral matching technique to properly label licensed users, we first consider a scenario with two primary users in the presence of noise and interference. The interference is included as a $10 \mathrm{~dB}$ pure tone at normalized frequency $\omega_{I}=0.375$. The primary user is assumed to be a BPSK signal with a rectangular pulse shape and 8 samples per symbol. The SNR of the desired users are $10 \mathrm{~dB}$ and $7 \mathrm{~dB}$, respectively, and their normalized carrier frequencies are $\omega_{1}=0.125$ and $\omega_{2}=0.625$, respectively. The spectral occupancy for this particular example is $\Omega=0.5$ (the primary users are using half of the available spectrum, i.e., $5 \mathrm{MHz}$ each of them). According to Landau's lower bound [57], the minimum average sampling rate for most signals is given by the Nyquist rate multiplied by the spectral occupancy, which is often much lower than the corresponding Nyquist rate. For this particular example, Landau's lower bound is telling us that half of the Nyquist samples can be thrown away without affecting the signal reconstruction. The values of $\xi_{u p}$ and $\xi_{\text {down }}$ for determining the stopping criterion of the WOMP are chosen $\xi_{\text {up }}=2$ and $\xi_{\text {down }}=0.5$.

Fig. 2 shows the result of the proposed detector for 5.000 Monte Carlo runs for different compression rates. Blue points indicate the output of the detector, black crosses represent the true primary users frequency-power representation and the red solid line indicates the interference frequency location. From Fig. 2 we observe that, in agreement with the Landau's lower bound, the proposed technique works well until the compression exceeds the limit given by the spectral occupancy. When the compression rate surpass $\rho=0.5$, the acquisition procedure loses too much information, which translates into a degradation of the detector performance. Fig. 3 shows the result of the proposed detector for 5.000 Monte Carlo runs for $\rho=1$ and $\rho=0.5$ when the interference is a QPSK signal. The spectral efficiency of QPSK is double that of BPSK, so its spectral shape is also a sync function but it occupies half of the spectrum that of the BPSK primary user (see Fig. 4). In Fig. 3, again, blue points indicate the output of the detector, black crosses represent the true primary users frequency-power representation and the red solid line indicates the interference frequency location. From the comparison of Fig. 2 and Fig. 3 it can be concluded that, as expected, the more similar the 


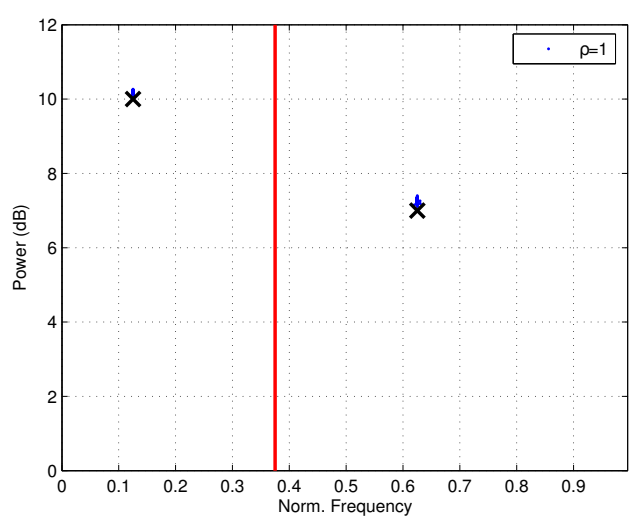

(a)

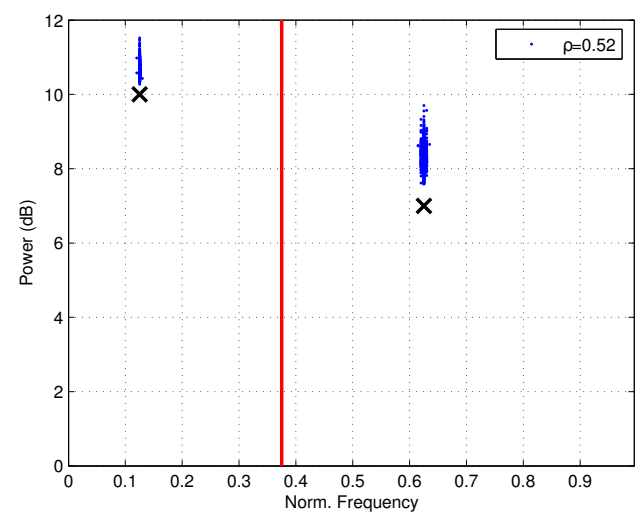

(c)

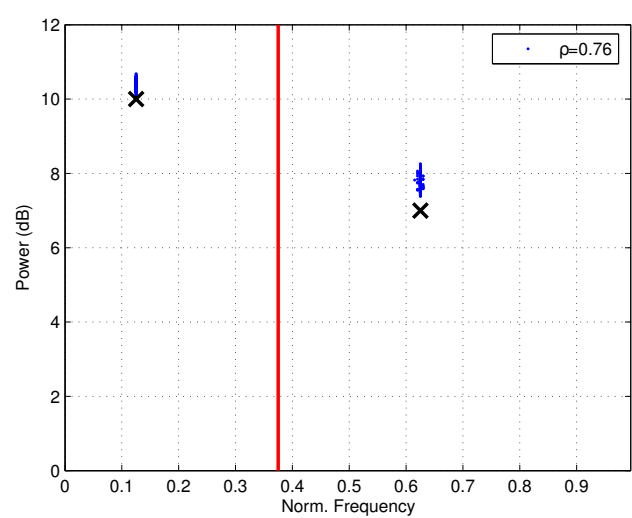

(b)

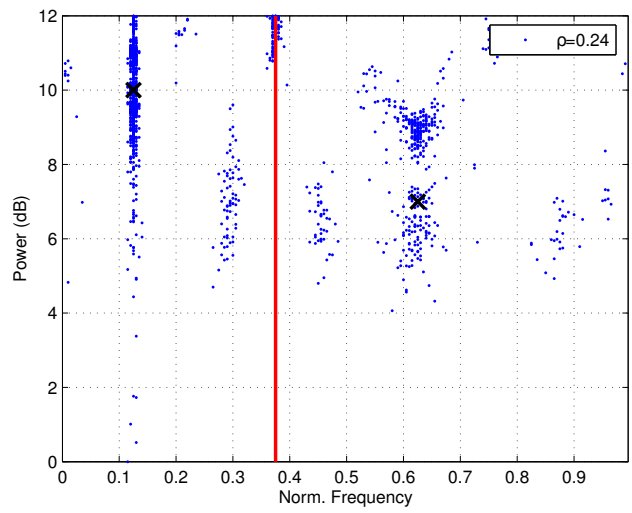

(d)

Fig. 2. Performance of the proposed CS-based spectral feature detector. Black crosses represent the true primary users frequency-power representation. A pure tone interference signal with SNR $=10 \mathrm{~dB}$ is located at $\omega_{I}=0.375$ (indicated by red solid line). (a) $\rho=1$, (b) $\rho=0.76$, (c) $\rho=0.52$, and (d) $\rho=0.24$.

interference and the primary signals are, the harder it is to discern them. However, it is also important to highlight that in the majority of the realizations, the proposed technique is able to discern the primary user from the interference. In particular, only in 3.5\% the algorithm gets confuse with the QPSK when considering half of the Nyquist samples.

The results obtained in Fig. 2 can be compared with the performance of the conventional periodogram spectral estimation. To this end, Fig. 5(a) shows the periodogram for the scenario under consideration for different compression rates. Besides presenting low resolution (becoming worse as the compression rate decreases), note also that they are not robust to the strong interference. In contrast, the proposed method provides a clear frequency and power estimate and makes the interference disappear because of their feature-based nature.

The interference rejection characteristic is linked to the reliability of the weights, which must provide a coarse estimate of the inverse transmitted power of the primary users. Fig. 5(b) shows the inverse of the weights for the scenario under consideration for different compression rates. As expected, the maxima of the inverse weights are located at the frequencies where a primary user is present and its value coincides with the primary users' SNR. However, the dynamic range of the weights significantly decrease when the compression rate surpass the limit of $\rho=0.5$, which explains the degradation suffered in Fig. 2(d).

For the evaluation of the frequency, power estimation and reconstruction accuracy, two types of scenario were considered. The first one with one active primary user with BPSK, using a rectangular pulse shape (with 8 samples per symbol) located at normalized frequency $\omega=0.5$ and with AWGN. The second one is the same as the first plus a pure tone interference with SNR $=10 \mathrm{~dB}$ located close to the primary at normalized frequency $\omega_{I}=0.6$. The noise is randomly generated among 5000 Monte Carlo iterations. Fig. 6(a) and Fig. 6(b) show the normalized RMSE of the estimated power level and the RMSE of the reconstruction error, respectively. The reconstruction error is computed using the spike trains distance defined in [58]. The amplitudes are first fixed to 1 and the resulting spike trains are independently convolved with $e^{-|\omega| / \Delta_{\omega}}$, producing slight spike spreading. Finally, the $l_{2}$-norm between the resulting convolved spike train and the convolved spike train corresponding to the real scenario is computed. The goal of this metric is to accurately represent perceptual differences between sparse signals. In general, the results displayed in Fig. 6 make evident that the presence of interference adversely affects the detector performance in terms of accuracy of the estimated parameters. As expected, the errors decrease as the sampling factor and the SNR increase. 


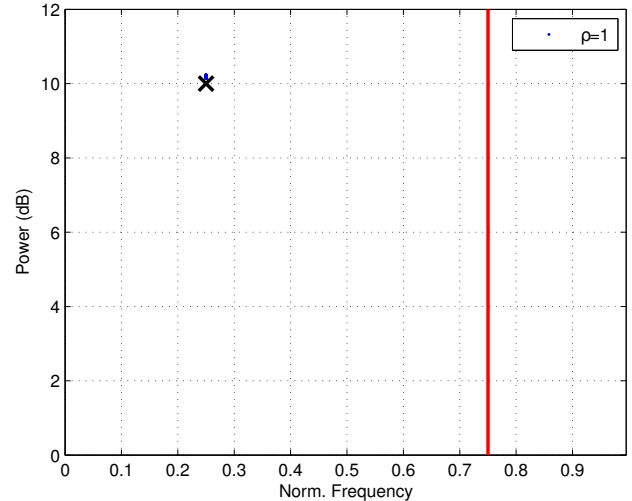

(a)

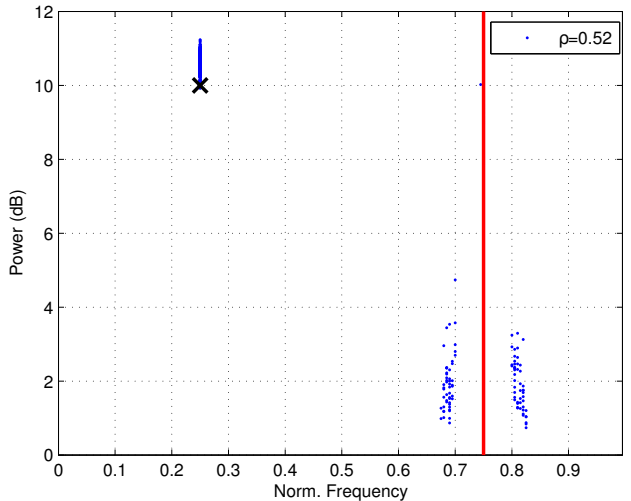

(b)

Fig. 3. Performance of the proposed CS-based spectral feature detector. Black crosses represent the true primary users frequency-power representation. A QPSK interference signal with SNR $=7 \mathrm{~dB}$ is located at $\omega_{I}=0.75$ (indicated by red solid line). (a) $\rho=1$, and (b) $\rho=0.52$.

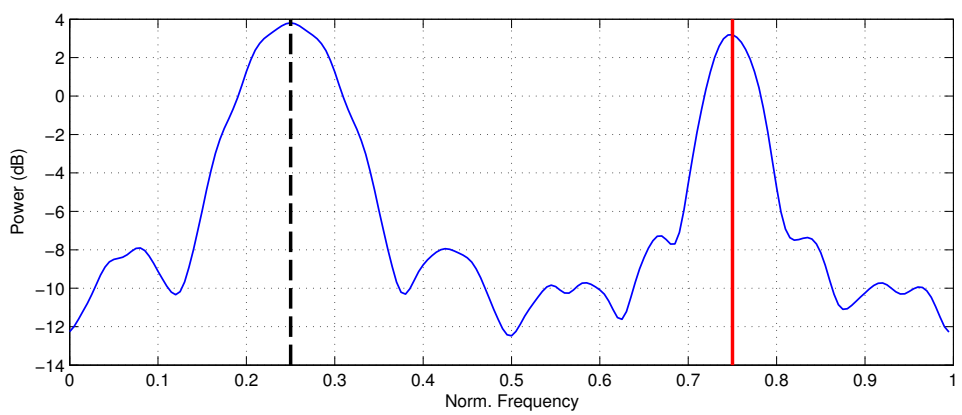

Fig. 4. Periodogram of the scenario considering a BPSK primary user located at normalized frequency $\omega=0.25$ with SNR $=10 \mathrm{~dB}$ and a QPSK interference signal located at normalized frequency $\omega=0.75$ with $\mathrm{SNR}=7 \mathrm{~dB}$.

High detection sensibility is a fundamental aspect in spectrum sensing for CR. In CS-based reconstruction, the stopping rule applied to halt the iterative reconstruction algorithm plays an important role in the detection performance. In particular, the interference rejection of the proposed detector is highly dependent on the value of $\xi_{u p}$ used in the stopping criterion defined in (33). To evaluate the probability of false alarm $\left(P_{f a}\right)$ with respect to $\xi_{\text {up }}$, we have run 1000 Monte Carlo iterations of an scenario consisting of a BPSK primary user located at normalized frequency $\omega=0.25$ and with $\mathrm{SNR}=0 \mathrm{~dB}$ and an interferent signal with the same SNR located at normalized frequency $\omega_{I}=0.75$. A false alarm occurs when the WOMP reconstruction algorithm selects something that is not the desired BPSK signal. In the whole simulation exercise we fixed $\xi_{\text {down }}=0.5$ to focus only on the parameter $\xi_{\text {up }}$. Fig. 7(a) shows the $P_{f a}$ versus $1+\xi_{u p}$ results for different compression rates when the interference is a pure tone. Note that values of $1+\xi_{\text {up }}$ close to one indicate very low interference rejection capabilities. As it can be appreciated from Fig. 7(a), the value of $\xi_{u p}$ that ensures low $P_{f a}$ is far away from zero when dealing with BPSKs and very narrow band interference. Fig. 7(b) shows the $P_{f a}$ versus $1+\xi_{u p}$ results when the interference is a QPSK modulated signal. The QPSK and the BPSK have similar spectral shape, which can lead to a high chance of miss-classification events. This fact is reflected in Fig. 7(b) with the values of $1+\xi_{\text {up }}$ that provide acceptable values of
$P_{f a}$, which are much lower than those shown in Fig. 7(a) for the pure tone interference.

Let us now evaluate the probability of detection $\left(P_{d}\right)$ versus SNR and versus $P_{f a}$. To do so, we consider three different scenario in which the primary user is a BPSK ( 8 samples per symbol) located at normalized frequency $\omega=0.25$. The first scenario considers the absence of interference signal, i.e., the primary is only contaminated with noise. The second and the third scenarios assume an interfering signal located at $\omega_{I}=0.25$, which is a pure tone with SNR $=10 \mathrm{~dB}$ and a QPSK with SNR $=7 \mathrm{~dB}$, respectively. To evaluate when the desired signal is correctly selected by the first iteration of the WOMP, we shall evaluate the selection step of the WOMP. At each iteration, the greedy selection chooses the atom that is most strongly correlated with the residual part of the signal, as shown in (31). In the first iteration, this is the atom that minimizes $\left\|\hat{\mathbf{r}}_{y}-\mathbf{A p}\right\|_{l_{2}}$. Thus, for the example at a hand, the function defined by $f\left(\omega_{m}\right)=\left|\left\langle\hat{\mathbf{r}}_{y}, \mathbf{a}_{m}\right\rangle\right| \cdot w_{m}^{-1}$ determines the detection performance. Usually, a threshold for $f\left(\omega_{m}\right)$ is chosen based on a given value of $P_{f a}$. Theoretical derivation of the threshold to meet the required $P_{f a}$ requires the statistical distribution of $f\left(\omega_{m}\right)$ for $\mathrm{H} 1$ and $\mathrm{H} 0$, which is a difficult task, especially when eigenvalues appear on the function $f\left(\omega_{m}\right)$. Since the main objective of the paper is to prove the spectral feature detector performance in sub-Nyquist spectrum sensing, as a first step, computer simulations will be 


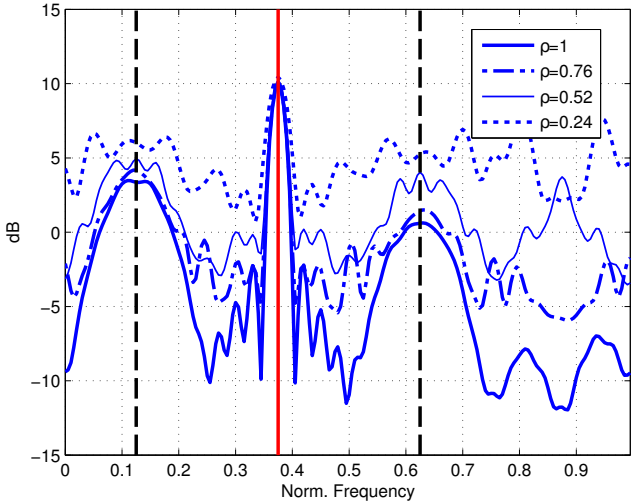

(a)

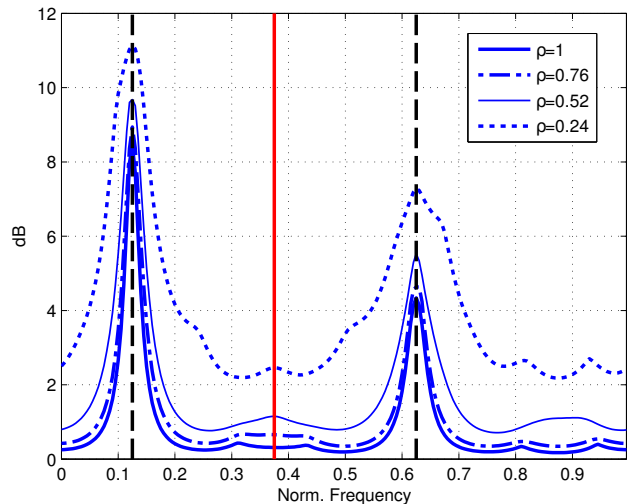

(b)

Fig. 5. The scenario considers two BPSK primary users ( $\mathrm{SNR}=10 \mathrm{~dB}$ and $\mathrm{SNR}=7 \mathrm{~dB}$ at $\omega_{1}=0.125$ and $\omega_{2}=0.625$, respectively) plus a pure tone interference signal ( $\mathrm{SNR}=10 \mathrm{~dB}$ at $\omega_{I}=0.375$ ): (a) Periodogram, (b) Inverse of weights.

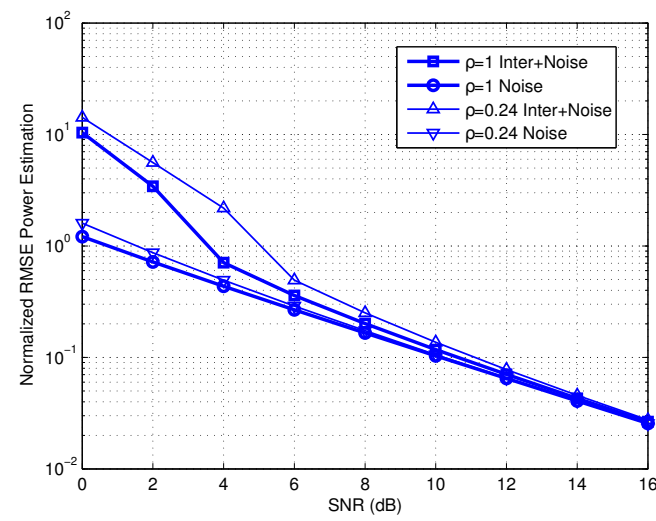

(a)

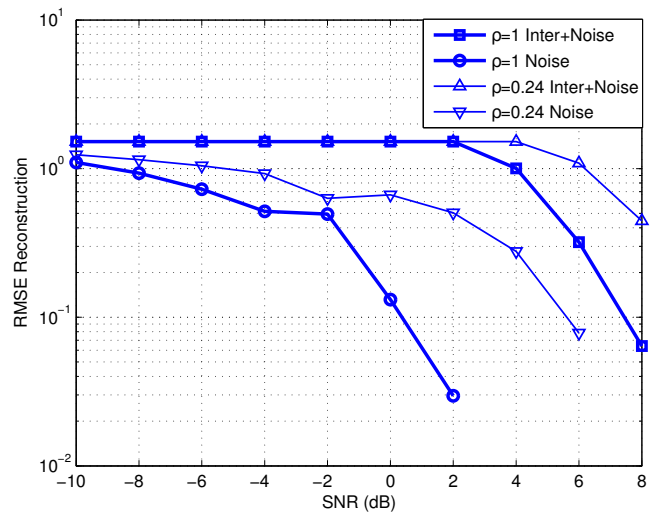

(b)

Fig. 6. Two types of scenario were considered. The first one with one active primary user with BPSK at $\omega=0.5$ plus AWGN. The second one is the same as the first plus a pure tone interference (SNR $=10 \mathrm{~dB}$ at $\omega_{I}=0.6$ ): (a) Normalized RMSE of the estimated power level, (b) RMSE of the reconstruction error.

used for estimating the statistical distribution of $f\left(\omega_{m}\right)$. To this end, we recorded 10.000 Monte Carlo simulations for H1 and $\mathrm{HO}$ and for all three scenarios described before, keeping the value of $f\left(\omega_{m}\right)$ of the true primary user frequency. Fig. 8 shows the obtained distributions of the function $f\left(\omega_{m}\right)$ for $\mathrm{H} 0$ and $\mathrm{H} 1$ for the first scenario with $\mathrm{SNR}=-18 \mathrm{~dB}$ and $\rho=0.76$. In Fig. 8 it is seen that both distributions closely match the Gaussian approximation (also shown in the figure with black solid line). In view of the results we have obtained, we decided to adopt Gaussian models for computing ROC (Receiver Operating Characteristic) figures. Therefore, from the 10.000 Monte Carlos runs we extract mean and variance of each hypothesis to build the corresponding probability density function (PDF) in the following way,

$$
\operatorname{pdf}(x)=\frac{1}{\sqrt{2 \pi \sigma_{x}^{2}}} e^{-\frac{\left(x-\mu_{x}\right)^{2}}{2 \sigma_{x}^{2}}}
$$

where $\mu_{x}$ and $\sigma_{x}^{2}$ denote the mean and the variance, respectively. In order to show the effect of the compression rate on the proposed detector, the ROC curves of the scenarios described above are compared in Fig. 9 for different compression rates and for different values of SNR. The curves show that the detector performance progressively deteriorate as $\rho$ and the SNR decreases. From Fig. 9(a), it can be observed that the proposed detector starts losing detection sensitivity around SNR $=-20 \mathrm{~dB}$, which goes inline with the sensitivity required by the IEEE 802.22 standard [59]. The dependence on the spectral similarities between the primary and the interference is evident by comparing Fig. 9(b) and Fig. 9(c), where the curves corresponding to a narrow band interference are shown to achieve better detection probabilities compared to those considering the relatively wider-band QPSK interference signal, even with lower values of SNR.

Fig. 10 shows the $P_{d}$ versus SNR results for the same scenario (a BPSK primary user located at $\omega=0.5$, free from interference) for a fixed probability of false alarm $P_{f a}=10^{-3}$. It is clear that, even for low compression rates, the proposed sparsity-based primary user detection approach is able to reliably detect very low primary transmission. For comparison, we plot in the same figure the results (no compression $\rho=1$ ) for the energy detection without noise uncertainty, the performance of the coarse estimate given by the inverse of the weights, and the two eigenvalue-based methods proposed in [21], namely Maximum-Minimum Eigenvalue (MME) and 


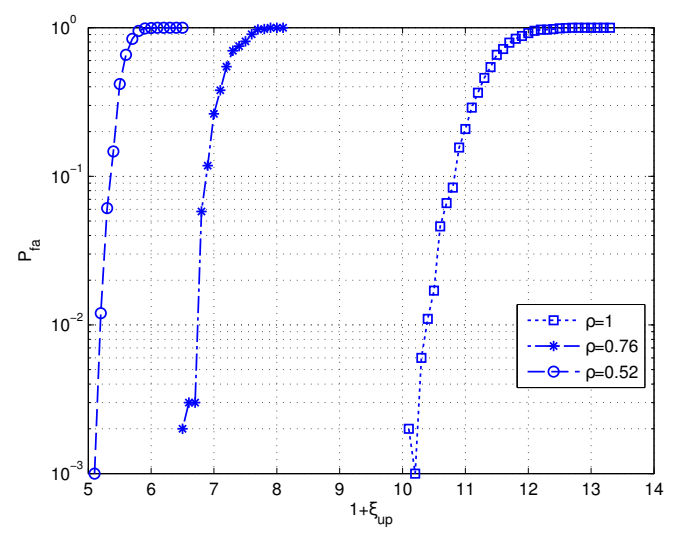

(a)

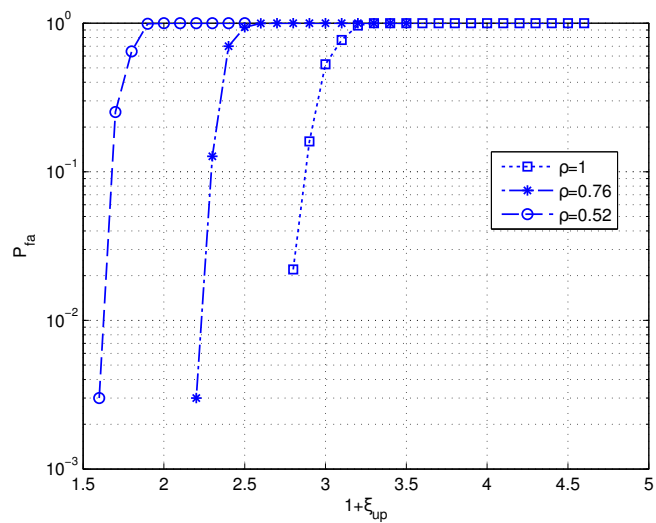

(b)

Fig. 7. Probability of false alarm versus $1+\xi_{\text {up }}$ for different compression rates. The scenario consists of a BPSK primary user (SNR $=0 \mathrm{~dB}$ at $\left.\omega=0.25\right)$ plus an interferent signal with the same SNR located at $\omega_{I}=0.75$. (a) Interference is pure tone, (b) Interference is QPSK.

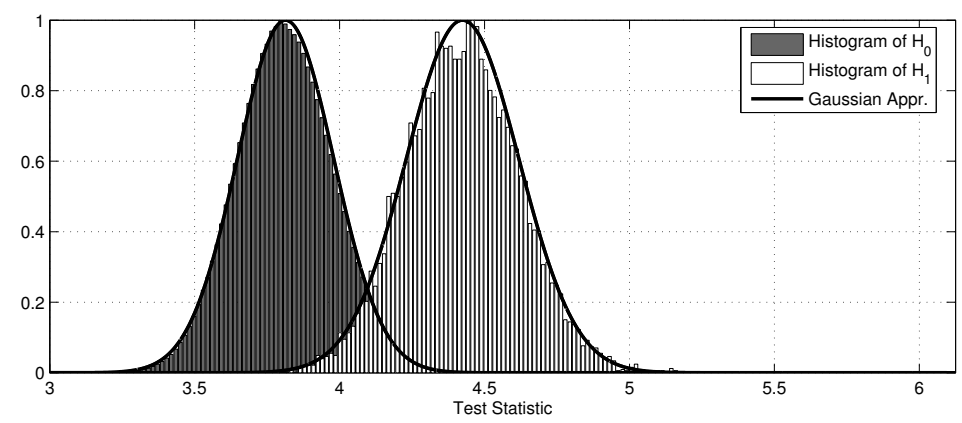

Fig. 8. Theoretical and empirical distributions of $f\left(\omega_{m}\right)$ for H1 and H0 for SNR $=-18 \mathrm{~dB}$ and $\rho=0.76$. The scenario for H1 consists of a BPSK primary user located at normalized frequency $\omega=0.5$ plus AWGN, and the scenario for H0 consists of only AWGN.

Energy with Minimum Eigenvalue (EME). As shown in the figure, the aforementioned competitors are much worse than that of the proposed technique. The reason is that the proposed technique makes use of signal features and exploits the sparse problem formulation. Let us now investigate the performance of the proposed detector with Ricean fading channel. We consider here that the signal propagates through 100 different 20-tap Rice channel realizations (a channel with a LOS and some random late arrivals). The LOS presents a gain of one, and the late random arrivals altogether also have energy equal to one. The performance is shown in Fig. 11 for different compression rates. The scenario consists of a BPSK primary user with 8 samples per symbol and SNR $=10 \mathrm{~dB}$ located at normalized frequency $\omega=0.25$ and a pure tone interference signal located at normalized frequency $\omega_{I}=0.75$ with $\mathrm{SNR}=10 \mathrm{~dB}$. In Fig. 11, blue points indicate the output of the detector, black cross represents the true primary user location and the red solid line indicates the interference location. It is seen that the method is robust in front of a frequency selective channel in terms of detection capabilities since the majority of detection events took place in close vicinity of the true primary user location. However, it is clear from Fig. 11 that the multipath causes quite small bias on the frequency and power estimation.

\section{CONCLUSION}

The performance of a CS-based spectral feature detector was proposed and evaluated in this paper. In particular, we take advantage of the sparsity described by the low spectral occupancy of the licensed radios not only to alleviate the sampling bottleneck but also to increase the accuracy of the detections by using sparsity-based recovery algorithms. An overcomplete dictionary that contains tunned spectral shapes of the primary user is proposed for sparse representation of the primary user spectral support, thus allowing the detection of primary users to be performed in a global unified optimization framework. The interference rejection is achieved by introducing weights to the $l_{1}$-norm and suppling a new stopping criterion for a conventional CS-based reconstruction method. Results based on computer simulations were presented, which showed the effectiveness in primary user detection and proved the interference rejection capabilities of the proposed method.

\section{REFERENCES}

[1] J. I. Mitola and G. J. Maguire, "Cognitive radio: making software radios more personal," IEEE Personal Communications, vol. 6, no. 4, pp. 1318, Aug, 1999.

[2] B. Wang and K. J. Ray Liu, "Advances in Cognitive Radio Networks: A Survey," IEEE Journal of Selected Topics in Signal Processing, vol. 5, pp. 5-23, 2011. 


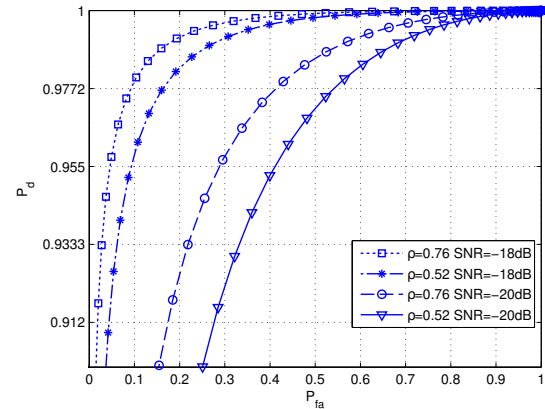

(a)

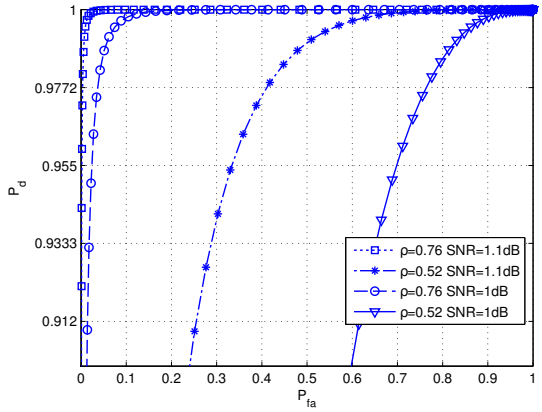

(b)

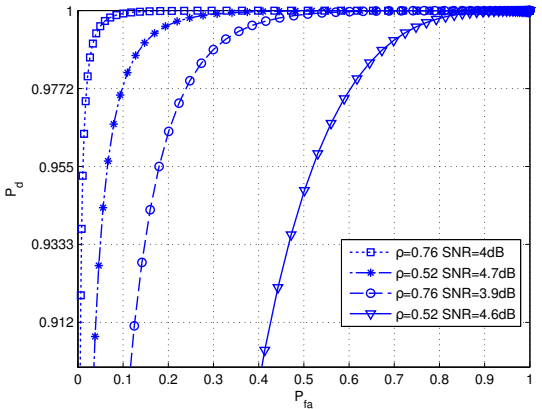

(c)

Fig. 9. ROC figures. The scenario considers a single desired signal which is a BPSK signal located at normalized frequency $\omega=0.25$ plus (a) noise, (b) noise plus a pure tone interferent signal $\left(\mathrm{SNR}=10 \mathrm{~dB}\right.$ at $\left.\omega_{I}=0.75\right)$, and (c) noise plus a QPSK interferent signal $\left(\mathrm{SNR}=7 \mathrm{~dB}\right.$ at $\left.\omega_{I}=0.75\right)$.

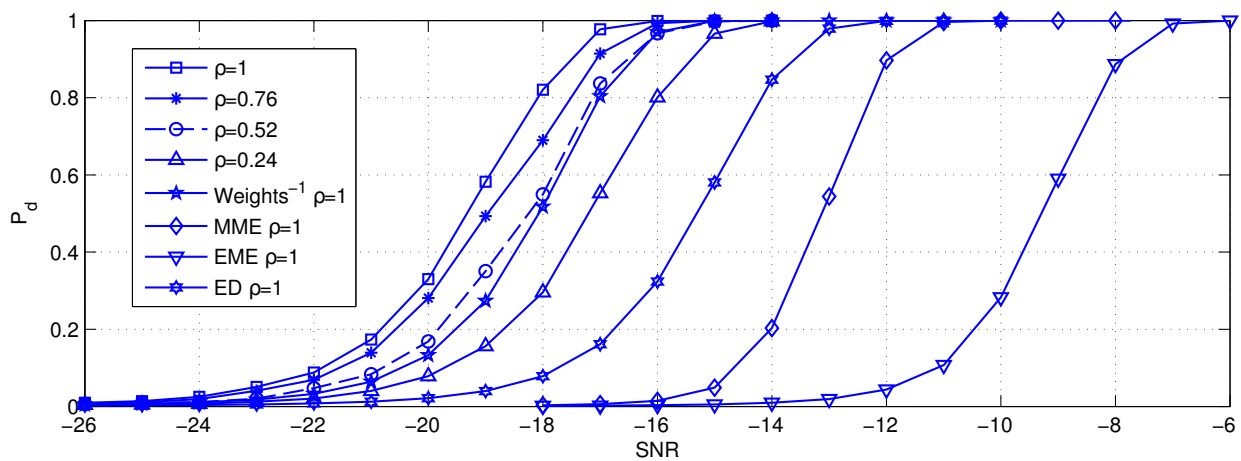

Fig. 10. Probability of detection versus SNR. The scenario considers a single desired signal which is a BPSK signal located at normalized frequency $\omega=0.5$.

[3] A. Goldsmith, S. A. Jafar, I. Maric, and S. Srinivasa, "Breaking Spectrum Gridlock with Cognitive Radio: An Information Theoretic Perspective," Proceedings of the IEEE, vol. 97, no. 5, pp. 894-914, May, 2009.

[4] F. C. Commission, "Tech. Report," TR 02-155, Nov, 2002.

[5] S. Haykin, "Cognitive radio: brain-empowered wireless communications," IEEE Journal on Selected Areas in Communications, vol. 23, no. 2, pp. 201-220, 2005.

[6] B. Fette, Cognitive Radio Technology. Communications Engineering Series, ELSEVIER, 2006

[7] E. Axell, G. Leus, E. Larsson, and V. Poor, "Spectrum Sensing for Cognitive Radio: State-of-the-Art and Recent Advances," IEEE Sig. Process. Magazine, vol. 29, no. 3, pp. 101-116, May, 2012.

[8] M. Kim and J. Takada, "Efficient Multichannel Wideband Spectrum Sensing Technique Using Filter Bank," IEEE Int. Symp. Pers. Indoor Mobile Radio Commun. (PIMRC), Tokyo, Japan, pp. 1014-1018, Sep, 2009.

[9] H. Sun, A. Nallanathan, C. X. Wang, and Y. Chen, "Wideband Spectrum Sensing for Cognitive Radio Networks: A Survey," Wireless Communications, IEEE, vol. 20, no. 2, pp. 74-81, Apr, 2013.

[10] D. Cabric, S. M. Mishra, and R. W. Brodersen, "Implementation issues in Spectrum Sensing for Cognitive Radios," Asilomar Conference on Signals, Systems and Computers, vol. 1, pp. 772-776, Nov, 2004.

[11] D. Sundman, S. Chatterjee, and M. Skoglund, "On the use of Compressive Sampling for Wide-Band Spectrum Sensing," IEEE Int. Sym. on Sig. Proc. and Inf. Tech., Luxor, Egypt, Dec, 2010.

[12] D. Donoho, "Compressed Sensing," IEEE Trans. Information Theory, vol. 52, no. 4, pp. 1289-1306, Apr, 2006.

[13] S. S. Chen, D. L. Donoho, and M. A. Saunders, "Atomic Decomposition by Basis Pursuit," SIAM J. on Sci. Comp., vol. 20, no. 1, pp. 33-61, 1998.

[14] Z. Tian and G. B. Giannakis, "Compressed Sensing for Wideband Cognitive Radios," International Conference on Acoustics, Speech and Signal Processing (ICASSP), Honolulu, HI, USA, Apr, 2007.

[15] M. Mishali and Y. Eldar, "From Theory to Practice: Sub-Nyquist Sampling of Sparse Wideband Analog Signals," IEEE J. Sel. Topics Signal Processing, vol. 4, no. 2, pp. 375-391, 2010.

[16] _ "Blind MultiBand Signal Reconstruction: Compressed Sensing for
Analog Signals," IEEE Trans. Signal Proces., vol. 57, no. 3, pp. 993 1009, 2009.

[17] C. Yen and X. Tsai, Y. Wang, "Wideband Spectrum Sensing Based on Sub-Nyquist Sampling," IEEE Trans. Sig. Process., vol. 61, no. 12, pp. 3028-3040, Jun, 2013.

[18] M. A. Lexa, M. E. Davies, J. S. Thompson, and J. Nikolic, "Compressive Power Spectral Density Estimation," Inter. Conf. on Acoustics, Speech and Sig. Process. (ICASSP), pp. 3884-3887, May, 2011.

[19] D. Ariananda and G. Leus, "Compressive Wideband Power Spectrum Estimation," IEEE Trans. Signal Process., pp. 4775-4788, Sep, 2012.

[20] H. Urkowitz, "Energy Detection of Unknown Deterministic Signals," Proceedings of the IEEE, vol. 55, pp. 523-531, Apr, 1967.

[21] Y. Zeng and Y. Liang, "Eigenvalue-Based Spectrum Sensing Algorithms for Cognitive Radio," IEEE Transactions on Communications, vol. 57, no. 6, pp. 1784-1793, Jun, 2009.

[22] M. Davenport, P. Boufounos, M. Wakin, and R. Baraniuk, "Signal Processing With Compressive Measurements," IEEE J. Sel. Topics Signal Process., vol. 4, no. 2, pp. 445-460, Apr, 2010.

[23] M. Aharon, "Overcomplete Dictionaries for Sparse Representation of Signals," Ph.D. thesis, Technion, Israel, Nov, 2006.

[24] Z. Quan, W. Zhang, S. Shellhammer, and A. Sayed, "Optimal Spectral Feature Detection for Spectrum Sensing at Very Low SNR," IEEE Trans. Commun., vol. 59, pp. 201-212, Jan, 2011.

[25] A. I. Pérez-Neira, M. A. Lagunas, M. A. Rojas, and P. Stoica, "Correlation Matching Approach for Spectrum Sensing in Open Spectrum Communications," IEEE Trans. Sig. Process., vol. 57, no. 12, pp. $4823-$ 4836, Dec, 2009.

[26] W. Gardner, A. Napolitano, and L. Paura, "Cyclostationarity: Half a century of Research," Elsevier Signal Processing, vol. 86, pp. 639-697, Apr, 2006.

[27] E. Lagunas and M. Najar, "Space-Time-Frequency Candidate Methods for Spectrum Sensing," 19th Europ. Sig. Process. Conf. (EUSIPCO), Aug, 2011.

[28] — "Compressive Spectrum Sensing Based on Spectral Feature Detection," Int. Symp. on Wireless Communication Systems (ISWCS), Ilmenau, Germany, Aug, 2013.

[29] — - "Sparse Correlation Matching-Based Spectrum Sensing for Open 


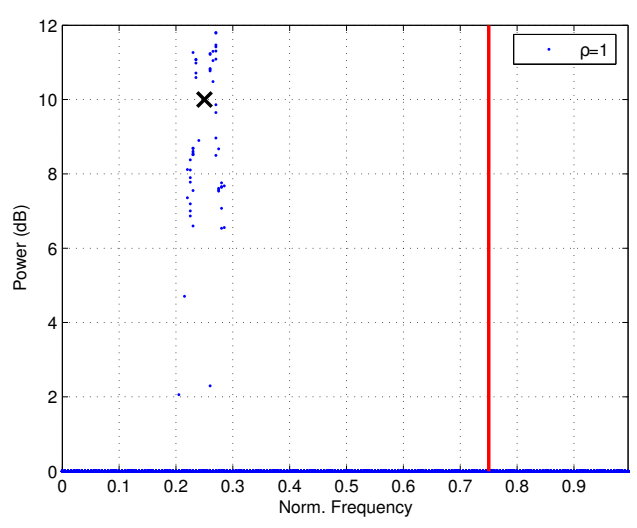

(a)

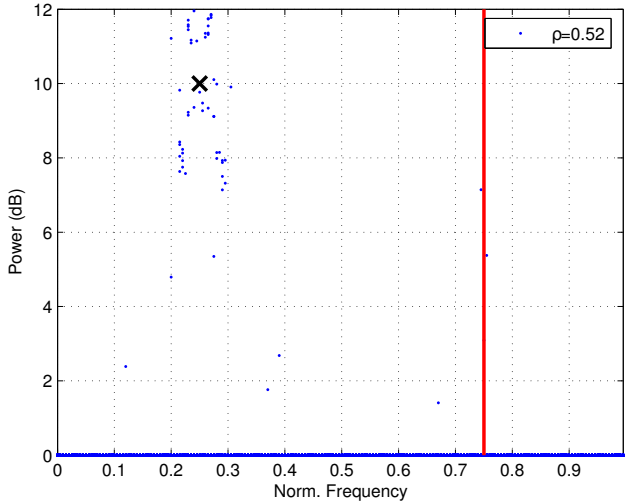

(b)

Fig. 11. Performance in a Rice Channel. The primary user is a BPSK ( $\mathrm{SNR}=10 \mathrm{~dB}$ at $\omega=0.25$ ). The interference is a pure tone located at $\omega_{I}=0.75$ with SNR $=10 \mathrm{~dB}$. 20-length Rice channel is considered. Here, $1+\xi_{\text {up }}=10$. (a) $\rho=1$, and (b) $\rho=0.52$.

Spectrum Communications," EURASIP Journal on Adv. in Sig. Process., 2012:31, Feb, 2012.

[30] P. Feng, "Universal Minimum-Rate Sampling and Spectrum-Blind Reconstruction for Multiband Signals," PhD, University of Illinois at Urbana-Champaign, USA, 1997.

[31] X. Zhai and J. Pan, "Energy-Detection Based Spectrum Sensing for Cognitive Radio," IET Conf. Wireless, Mobile and Sensor Networks (CCWMSN07), pp. 944-947, 2007.

[32] O. Holland, P. Cordier, M. Muck, L. Mazet, C. Klock, and T. Renk, "Spectrum Power Measurements in 2G and 3G Cellular Phone Bands During the 2006 Football World Cup in Germany," IEEE Symp. on New Frontiers in Dynamic Spectrum Access Networks (DySPAN), Dublin, Ireland, Apr, 2007.

[33] M. A. McHenry, "NSF Spectrum Occupancy Measurements Project Summary," Shared Spectrum Company, Tech Report, Aug, 2005.

[34] D. Romero and G. Leus, "Compressive Covariance Sampling," Information Theory and Applications Workshop (ITA), San Diego, CA, USA, Feb, 2013.

[35] P. Feng and Y. Bresler, "Spectrum-blind Minimum-rate Sampling and Reconstruction of Multiband Signals," Inter. Conf. on Acoustics, Speech, and Signal Processing, vol. 3, pp. 1688-1691, 1996.

[36] R. Venkataramani and Y. Bresler, "Optimal Sub-Nyquist Nonuniform Sampling and Reconstruction for Multiband Signals," IEEE Sig. Process. Letters, vol. 18, no. 8, pp. 443-446, Aug, 2011.

[37] H. Sun, W. Chiu, J. Jiang, A. Nallanathan, and H. Poor, "Wideband Spectrum Sensing With Sub-Nyquist Sampling in Cognitive Radios," IEEE Trans. Sig. Process., vol. 60, no. 11, pp. 6068-6073, Nov, 2012.

[38] S. Boyd and L. Vandenberghe, Convex Optimization. Cambridge University Press, 2004.

[39] E. Candes, M. Wakin, and S. Boyd, "Enhancing Sparsity by Reweighted $l_{1}$ Minimization," Journal Fourier Analysis and Applications, vol. 14, no. 5-6, pp. 877-905, Oct, 2008.

[40] J. A. Tropp and A. C. Gilbert, "Signal Recovery From Random Measurements Via Orthogonal Matching Pursuit," IEEE Transactions on Information Theory, vol. 53, no. 12, pp. 4655-4666, Dec, 2007.

[41] R. Tibshirani, "Regression Shrinkage and Selection via the Lasso," Journal of the Royal Statistical Society, vol. 58, no. 1, pp. 267-288, 1996.

[42] J. Tropp and D. Needell, "CoSaMP: Iterative Signal Recovery from Incomplete and Inaccurate Samples," Applied and Computational Harmonic Analysis, vol. 26, no. 3, pp. 301-321, Apr, 2008.

[43] E. J. Candes, J. Romberg, and T. Tao, "Stable Signal Recovery from Incomplete and Inaccurate Measurements," Comm. on Pure and Applied Mathematics, vol. 59, no. 8, pp. 1207-1223, Aug, 2006.

[44] Z. Wang, G. Arce, B. Sadler, J. Paredes, S. Hoyos, and Z. Yu, "Compressed UWB Signal Detection with Narrowband Interference Mitigation," IEEE Int. Conf. UWB (ICUWB), Sep, 2008.

[45] J. Laska, M. Davenport, and R. Baraniuk, "Exact Signal Recovery from Sparsely Corrupted Measurements Through the Pursuit of Justice," Asilomar Conf., Nov, 2009.

[46] M. Davenport, P. Boufounos, and R. Baraniuk, "Compressive Domain
Interference Cancellation," Signal Processing with Adaptive Sparse Structured Representations (SARS), Mar, 2009.

[47] E. J. Candes and T. Tao, "The Dantzig Selector: Statistical Estimation when $\mathrm{p}$ is much larger than n," Annals of Statistics, vol. 35, no. 6, pp. 2313-2351, Dec, 2007.

[48] J. Haupt and R. Nowak, "Signal Reconstruction from Noisy Random Projections," IEEE Transactions on Information Theory, vol. 52, no. 9, pp. 4036-4048, 2006.

[49] S. G. Mallat and Z. Zhang, "Matching Pursuits with Time-Frequency Dictionaries," IEEE Transactions on Signal Processing, vol. 41, no. 12, pp. 3397-3415, Dec, 1993.

[50] J. Tropp, A. Gilbert, and M. Strauss, "Algorithms for Simultaneous Sparse Approximation: Part I: Greedy Pursuit," Signal Process., vol. 86, no. 3, pp. 572-588, Mar, 2006.

[51] P. Boufounos, M. Duarte, and R. Baraniuk, "Sparse Signal Reconstruction from Noisy Compressive Measurements using Cross Validation," IEEE Stat. Sig. Proces. Workshop, Madison, WI, USA, pp. 299-303, Aug, 2007.

[52] M. Lopes, "Estimating Unknown Sparsity in Compressed Sensing," JMLR Int. Conf. on Machine Learning, vol. 28, no. 3, pp. 217-225, Feb, 2013.

[53] O. Escoda, L. Granai, and P. Vandergheynst, "On the Use of a Priori Information for Sparse Signal Approximations," IEEE Trans. Sig. Process., vol. 54, no. 9, pp. 3468-3482, Sep, 2006.

[54] Z. Ye, G. Memik, and J. Grosspietsch, "Energy Detection Using Estimated Noise Variance for Spectrum Sensing in Cognitive Radio Networks," IEEE Conf. on Wireless Communications and Networking, Las Vegas, NV, USA, Mar, 2008.

[55] J. Shen, S. Liu, and Y. Wang, "Robust Energy Detection in Cognitive Radio," IET Commun., vol. 3, no. 6, pp. 1016-1023, 2009.

[56] J. Li, W. Zhang, and J. Yuan, "Opportunistic Spectrum Sharing in Cognitive Radio Networks Based on Primary Limited Feedback," IEEE Trans. on Communications, vol. 59, no. 12, pp. 3272-3277, Dec, 2011.

[57] H. Landau, "Necessary Density Conditions for Sampling and Interpolation of Certain Entire Functions," Acta Math., vol. 117, no. 81, pp. 37-52, 1967.

[58] M. van Rossum, "A novel spike distance," Neural Computation, vol. 13, no. 4, pp. 751-763, Apr, 2001.

[59] S. J. Shellhammer, "Spectrum Sensing in the IEEE 802.22," IAPR Workshop on Cognitive Information Processing, Santorini, Greece, pp. 1-6, Jun, 2008. 


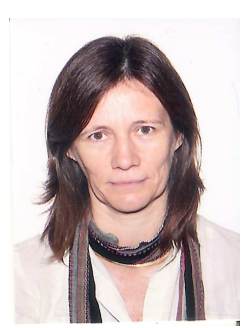

Montse Nájar received the Electrical Engineering degree and the $\mathrm{Ph} \mathrm{D}$. from the Polytechnic University of Catalonia (UPC), Barcelona, Spain, in 1991 and 1996, respectively. In 1992, she joined the Department of Signal Theory and Communication of the UPC. Since 1997 she is an Associate Professor at the UPC, where she teaches and coordinates undergraduate and graduate courses in Digital Communications and Signal Processing. From 2003 to 2006 she was member of the Board of Directors of the Telecommunications School of Barcelona, ETSETB. From 2005 to 2013 she was research associate in the CTTC (Centre Tecnologic de Telecomunicacions de Catalunya). Her current research interests include signal processing with application to communication systems, array signal processing and location in wireless systems. She has participated in several IST and Eureka European projects as well as national public and private funded projects. She has been guest editor of the EURASIP Signal Processing journal. She is reviewer of the IEEE and the EURASIP Signal Processing journals.
Eva Lagunas (S'09-M'13) received the M.Sc. and $\mathrm{Ph} . \mathrm{D}$. degrees in telecommunications engineering from the Polytechnic University of Catalonia (UPC), Barcelona, Spain, in 2010 and 2014, respectively. She was awarded with a scholarship of the Catalan Government Grant (FI-DGR) to carry her PhD studies. She was Research Assistant within the Department of Signal Theory and Communications, UPC, from 2009 to 2013. During the summer of 2009 she was a guest research assistant within the Department of Information Engineering, Pisa, Italy, developing TOA estimation algorithms in UWB systems. From November 2011 to May 2012 she held a visiting research appointment at the Center for Advanced Communications (CAC), Villanova University, PA, USA, where she worked on through-wall radar imaging technology. In September 2014, she joined the Interdisciplinary Centre for Security, Reliability and Trust (SnT), University of Luxembourg, where she works on Cognitive Satellite Communications. 